\title{
The NORMAN Network and its activities on emerging environmental substances with a focus on effect-directed analysis of complex environmental contamination
}

\author{
Werner Brack ${ }^{1 *}$, Valeria Dulio ${ }^{2}$ and Jaroslav Slobodnik ${ }^{3}$
}

\begin{abstract}
The need to look beyond the conventional target pollutants when assessing the hazards of chemicals to human health and to ecosystems is now generally recognised as a priority issue in all environmental policy areas at both the European level and national level in the various countries. It has also become clear that it is not possible for individual countries alone to develop the knowledge and methodologies needed for measuring and evaluating the effects and associated risks of a vast number of emerging pollutants. Further to these priority needs, the NORMAN project (www.norman-network.net) was funded in 2005 by the European Commission in order to promote the creation of a permanent network among reference laboratories and research centres, in collaboration with the parties involved (industry, standardisation bodies, NGOs, etc.), to ensure (i) a more rapid and wide-scope exchange of data and information on the occurrence and effects of emerging substances, (ii) better data quality and comparability via validation and harmonisation of common measurement methods (chemical and biological) and monitoring tools, (iii) more transparent information (need for information, not just data) and (iv) the establishment of an independent and competent forum for the technical/scientific debate on issues related to emerging substances. NORMAN plays a significant role as an interface organisation between science and policy, with the advantage of speaking with a "bigger voice" to the European Commission and other public institutions. The activities of the network range from a scientific watch and the feeding of data on emerging substances into NORMAN databases (information gateway on emerging pollutants) to the organisation of working groups and workshops (producing position papers on research priorities), the setting-up of interlaboratory studies and the organisation of measurement campaigns. This article presents the objectives and scope of the activities of the NORMAN network, together with a summary of its concrete achievements after six years of existence. Moreover, the article gives a special insight in the work done by the NORMAN Working Group on effect-directed analysis for the identification of hazardous pollutants.
\end{abstract}

Keywords: Emerging substances, Effect-directed analysis, Monitoring

\section{Background}

The need to look beyond the traditional target pollutants when assessing the hazards of chemicals to human health and to ecosystems is now generally recognised as a priority issue in all policy areas at the national, European and world level. "Emerging substances", i.e. "substances of

\footnotetext{
* Correspondence: werner.brack@ufz.de

${ }^{1}$ Helmholtz Centre for Environmental Research - UFZ, Leipzig, Germany

Full list of author information is available at the end of the article
}

emerging concern", are therefore a field of increasing interest in environmental research and monitoring.

There are numerous target substances and it is impossible to develop the necessary knowledge and methodologies solely at the national scale.

Moreover, regarding data quality and validation, in particular with respect to emerging pollutants, the lack of harmonisation in method validation and QA/QC requirements is a cause for concern over the reliability of analytical data. 
Finally, today we still lack the capacity for systematic early recognition of those substances that should be addressed as "relevant" emerging toxicants in a European context, as opposed to those 'believed' to be emerging.

So, there is a need for an information exchange on emerging substances that is officially recognised by the authorities at the European level, and beyond, in order to advance knowledge of their impacts and to support decision-making.

The NORMAN network (www.norman-network.net) was created in 2005 in response to a DG Research call for projects under the 6th Framework Programme. The aim of the project was to set up a permanent network of reference laboratories, research centres and related organisations in order to facilitate the exchange of information on emerging substances and to improve data quality and validation of measurement methods.

In November 2008, when the European Commission (EC) contract and funding expired, NORMAN - consisting of 17 partners led by INERIS - was duly set up as a permanent, self-sustaining network (non-profit association) of reference laboratories, research centres and related organisations for the monitoring and bio-monitoring of emerging environmental substances. The NORMAN network today has 50 members [1] from 19 countries (including two members from Canada) and it is now a functioning and well recognised network, thanks to members' financial and in-kind contributions and the achievement of successful synergies between research teams in the field of emerging substances.

\section{Mission and scope of activities of the NORMAN Network}

The mission of the NORMAN network is to enhance the exchange of information and collection of data on emerging environmental substances and to encourage validation and harmonisation of measurement methods and monitoring tools so that the demands of risk assessors can be better met.

The activities of the NORMAN network address emerging contaminants in all environmental compartments, covering all steps, from first identification of a potential new emerging substance based on evidence of occurrence in the environment and / or identification of effects on the ecosystems and human health, down to confirmation of its status as a future regulated priority pollutant. In this context, the network strongly supports an integrated approach, including chemical and biological tools, for the identification of substances that should be considered for further investigation, either at the local scale or more widely.

NORMAN plays a significant role as an interface organisation between science and policy, with the advantage of speaking with a "bigger voice" to the EC and other public institutions. The activities of the network range from a scientific watch and the feeding of data on emerging substances into the NORMAN databases (information gateway on emerging pollutants) to the organisation of working groups and workshops (producing position papers on research priorities), the setting-up of interlaboratory studies and the organisation of measurement campaigns.

\section{NORMAN's achievements and outputs at the end of 2011}

Among the activities to be highlighted: two working groups have been operational since 2010. The WG on Prioritisation of Emerging Substances identifies emerging substances for priority attention (including priority needs for improving existing data, analytical methods, biological tests, etc.). With regard to the specific needs of emerging substances, NORMAN has developed an improved prioritisation methodology [2], which has already been published and applied to an independent dataset of the MODELKEY project [3]. In general, the added value of the NORMAN methodology, compared to the previous prioritisation approaches, is that it is a global scheme which also considers compounds for which not enough data are available and prioritises them by action needed, taking into account the current knowledge gaps. A second WG, on effect-directed analysis (EDA) for hazardous pollutant identification, brings together major European experts in EDA to promote scientific progress in this research field, to identify hazardous compounds in support of current prioritisation processes. A WG on bioassays and biomarkers linked to the EDA WG will be part of the network's activities in 2012 to look at current applications of biological test tools in water quality monitoring. Finally, a WG on engineered nanoparticles (ENP) was officially established in October 2011 to address: 1) behaviour of ENPs in the aquatic environment (wastewater, storm water, surface water and sediment); 2) their effects on aquatic ecosystems and 3) analytical methods.

Events held by the NORMAN network include: a workshop on Mixtures and Metabolites of Chemicals of Emerging Concern, co-organised in Amsterdam by RIVM and IVM (November 2009), which considered the metabolites and degradation products needing further investigation and monitoring for a more complete picture of the impact of chemical compounds; a workshop on Selection and Monitoring of River Basin-Specific Pollutants in EU Member States [4], organised by JRC-IES (10-11 June 2010), where Member States were invited to exchange information on current approaches for identification and monitoring of River Basin-Specific Pollutants (including emerging pollutants), as required by the Water Framework Directive; a workshop on Environmental Specimen 
Banking (ESB) [5] organised in Berlin (20-21 June 2010) by the German UBA, to share experiences and explore the potential for closer networking of ESBs and for cooperating with EU chemical safety management; a workshop on "new" brominated flame retardants, their occurrence in the environment and their effects, organised by IVL in Stockholm (23-24 November 2011); and finally a workshop on challenges in sampling and analysis of emerging contaminants, organised by NIVA (Norway) in Oslo, 1-2 March 2012.

Further events in the NORMAN Programme of Activities for 2012 include: 1) a workshop on micropollutants, metabolites and mixtures in drinking water and its sources, co-organised by IWW (Germany) and KWR (The Netherlands); 2) a workshop held jointly by the NORMAN Network, COST Action TD0803 (DARE) and the NIREAS-International Water Research Center (Cyprus, 13-14 September) to discuss the challenges of wastewater reuse practices with regard to contaminants of emerging concern, their transformation while in the environment, their potential uptake by plants and crops and their effects; and 3) a workshop on occurrence, fate and effects of emerging pollutants in the environment, organised by IVM (The Netherlands), which will take place in Amsterdam on 29-30 November 2012.

Since its creation as an EU-funded project, NORMAN has been maintaining and regularly feeding three webbased databases: (i) EMPOMAP [6]: a database of leading experts, organisations and projects dealing with emerging substances; (ii) EMPODAT [7]: a database of geo-referenced monitoring and occurrence data accompanied by ecotoxicological information from bioassays; and (iii) NORMAN MassBank (former EMPOMASS): a database of mass spectrometric information on provisionally identified and unknown substances. As to the latter, the former EMPOMASS database was transferred in 2011 to the new MassBank portal [8] (hosted by UFZ, Leipzig, Germany). It is now available on the NORMAN public site as a platform for the collection and exchange of mass spectral data among NORMAN members and between NORMAN and the scientific and regulatory community worldwide. With this initiative the NORMAN network intends to contribute to motivating the environmental chemistry community to share their mass spectra of analytical standards and environmental samples with others in order to improve the identification of environmental suspects and unknowns.

As regards the methods validation aspects, particularly in respect of emerging pollutants, there is concern about the reliability of analytical data, due to a lack of harmonisation in method validation and $\mathrm{QA} / \mathrm{QC}$ requirements. A major achievement of the NORMAN experts was the publication in 2009 of a common European protocol for the validation of methods $[9,10]$ for the monitoring (occurrence) and bio-monitoring (effects) of emerging pollutants in environmental matrices. The protocol has been tested in three case studies as part of the NORMAN project, and negotiations are now under way at CEN for the adoption of this protocol as CEN Technical Specifications. Moreover, based on the identification of needs for specific emerging substances or groups of substances, NORMAN encourages Proficiency Testing (PT) providers and / or partners of the network to organise and conduct interlaboratory studies. In 2011, further to the publication of a Position Paper of the NORMAN Expert Group on Passive Sampling [11], an international intercalibration study on passive sampling was organised by NORMAN (Water Research Institute, Slovakia) in collaboration with the JRC-IES of the European Commission. An important critical point in the application of passive samplers in water quality monitoring is their compatibility with the requirements of the Water Framework Directive (WFD). It is therefore envisaged that the dissemination workshop, which will take place in 2012, should be organised in connection with a NORMAN Expert Group meeting with invited experts in ecotoxicology and in passive sampling (analytical aspects) to start discussion about the possible routes to match the Environmental Quality Standards (EQS) established under the WFD with passive sampling results.

Other recent examples of interlaboratory studies organised by NORMAN are: 1) a study of perfluorinated alkyl substances in environmental samples [12], i.e. surface water, marine fish and sludge (IVM in collaboration with QUASIMEME in 2009); 2) the on-going exercise on analysis of organic phosphorous flame retardants (PFR) in environmental and dust samples, aimed at the transfer of knowledge on PFR analysis between laboratories, and assessment of how well the laboratories perform (IVM, 2011).

\section{Effect-directed analysis for identification of relevant emerging contaminants in complex environmental samples}

There is increasing concern about the fact that environmental samples typically contain tens of thousands of individual chemicals, most of which are unknown and not characterised for potential adverse effects. Since a full analysis and assessment of these compounds will not be possible, EDA is regarded as a potential tool to isolate, identify and prioritise those chemicals which cause measurable effects on biological systems. Since the identification of emerging substances of toxicological concern is a key objective of NORMAN, a new NORMAN WG on EDA was created in 2011 with the mission of bringing together European experts in EDA to exchange knowledge, methods and data via workshops, data sharing and scientific collaboration, to compile existing EDA 
methodologies for scientists and end-users and to promote scientific progress in, and application of, EDA tools for a more realistic quality assessment of complex contaminated environments.

The NORMAN WG on EDA identified several gaps that hamper the broad and successful application of EDA for the identification of new toxicants in the environment. These gaps include a comprehensive compilation of tools and approaches in EDA for scientists and practitioners, a common mass spectra database particularly for LC-MS/MS data, and interdisciplinary-trained young scientists in the field of EDA. Thus, the first major contributions of the NORMAN EDA WG to close these gaps were: (i) the publication of a handbook on EDA providing an overview of important approaches and techniques to relate observed effects to causing chemicals [13], (ii) the creation of NORMAN MassBank as a tool to support the analytical identification of emerging pollutants all over Europe, and (iii) the launch of a new Marie Curie Action - Initial Training Network (ITN) called EDA-EMERGE, with 14 partners all over Europe.

The new Handbook of Environmental Chemistry on Effect-Directed Analysis of Complex Environmental Contamination [13] compiles contributions of NORMAN members and outstanding scientists beyond the network, and is designed as a comprehensive and interdisciplinary source of information for environmental scientists and environmental agencies dealing with the analysis, monitoring and assessment of environmental contamination. Major topics include the development of Toxicity Identification Evaluation (TIE) in the U.S. and of EDA in Europe, separation techniques and advanced GC-MS, LC-MS and computer tools for structure elucidation in EDA, simultaneous screening and chemical characterisation of bioactive compounds using affinity chromatography, biodiagnostic tools for mutagens, endocrine disruptors and AhR-mediated toxicants, and approaches to confirm the ecological relevance of identified key toxicants in aquatic systems.

The web-based MassBank as a community-driven open source approach for peak deconvolution, mass spectral evaluation and library searches, has been established by the metabolomics community [14] and is now adapted to environmental contaminants by the NORMAN EDA WG [15]. In the NORMAN MassBank, mass spectral records from different instrument types, ionisation techniques and collision energies obtained from standards and environmental samples provided by NORMAN members are included. MassBank offers sophisticated, vendor-independent storage and search options for any kind of high and low resolution mass spectra including, for example, EI-MS, ToF-MS and FT-MS. MassBank was recently combined with the in silico fragmentation tool MetFrag [16] (http://msbi.ipb-halle.de/
MetFrag) to build up MetFusion (http://msbi.ipb-halle/ MetFusion) and linked as a database search option within MzMine (available from version 2.5). This approach helps to overcome problems with different instruments and/or experimental settings, and supports the identification of unknown and emerging compounds using conventional and modern mass spectrometers coupled to gas or liquid chromatography. NORMAN members and the environmental and analytical community beyond NORMAN are invited to share their mass spectra of analytical standards and environmental samples with others to improve the identification of emerging toxicants in the environment.

The NORMAN-based ITN EDA-EMERGE aims to train a new generation of young scientists in the interdisciplinary techniques required to identify emerging pollutants affecting biological systems in aquatic environments. This will meet major challenges in the monitoring, assessment and management of toxic pollution in European river basins considering the enormous complexity of contamination, effects and cause-effect relationships. By integrating innovative mode-of-action-based biodiagnostic tools including in vitro tests, transgenic organisms and "omics" techniques with powerful fractionation and cutting edge analytical and computational structure elucidation tools, a new generation of effect directed analysis (EDA) approaches will be developed for the identification of toxicants in European surface and drinking waters. Innovative method development by young researchers at universities, research centres and private companies is interlinked with a joint European demonstration programme and higher tier EDA, and extensive training courses. A first milestone will be the EDA-EMERGE summer school at the UFZ in Leipzig, Germany (25-29 July), which provides PhD students from EDA-EMERGE, NORMAN and beyond with the basics for successful EDA in aquatic systems.

To further continue the fruitful work of this NORMAN WG the already-mentioned workshop on "Occurrence, fate and effects of emerging pollutants in the environment - chemical analysis and toxicological assessment" will be organised by the Institute for Environmental Studies at the Free University of Amsterdam on 29-30 November in Amsterdam (http://www.normannetwork.net/). Major topics include the occurrence and fate of new compound classes, non-target analysis and identification strategies, biological tools for effect assessment and effect-directed analysis. In addition, an indepth introduction to the use of MassBank, including practical exercises, will be provided.

\section{Conclusions}

NORMAN provides a valuable and permanent network of research institutions, reference laboratories and 
stakeholders to support decision-makers in all decisions for the identification, monitoring, assessment and prioritisation of relevant emerging pollutants. While this concerns a large number of known but unregulated pollutants and significant data gaps, NORMAN also makes efforts to identify so far unknown chemicals with potentially adverse effects. In a typical environmental sample, represented by a gas or liquid chromatogramme, unknowns are still the majority and only joint efforts of the scientific community will help to change this situation. NORMAN and its WGs offer their expertise and facilities to organise and structure this process. Thus, joining this network and becoming actively involved in the multiple activities of its WGs is not only beneficial for the individual scientist, but also helps to solve the great challenges related to emerging pollutants at a local, national, European and global scale, and supports the integration of state-of-the-art science into regulation, monitoring and management of the environment.

\section{Competing interests}

The authors declare that they have no competing interests.

\section{Authors' contributions}

All authors have read and approved the final manuscript.

\section{Author details}

${ }^{1}$ Helmholtz Centre for Environmental Research - UFZ, Leipzig, Germany.

${ }^{2}$ INERIS, National Institute for Environment and Industrial Risks,

Verneuil-en-Halatte, France. ${ }^{3}$ Environmental Institute, Kos, Slovak Republic.

Received: 29 August 2012 Accepted: 29 August 2012

Published: 17 October 2012

\section{References}

1. List of NORMAN members. [http://www.norman-network.net/index_php. php?module=public/about_us/permanent_networkb\&partie_texte= members_norman\&menu2=public/about_us/about_us].

2. Dulio V, von der Ohe PC, Slobodnik J, NORMAN Prioritisation WG: The NORMAN approach for setting priorities among emerging contaminants in Europe: Working Group 1. NORMAN; 2011. http://www.norman-network.net/index_php.php.

3. von der Ohe PC, Dulio V, Slobodnik J, De Deckere E, Kuhne R, Ebert RU, Ginebreda A, De Cooman W, Schuurmann G, Brack W: A new risk assessment approach for the prioritization of 500 classical and emerging organic microcontaminants as potential river basin specific pollutants under the European Water Framework Directive. Sci Total Environ 2011, 409:2064-2077.

4. Piha H, Dulio V, Hanke G: Workshop report: River basin-specific pollutants identification and monitoring. Luxembourg: Publications Office of the European Union; 2010:59

5. Koschorreck J: Conference for european environmental specimen banks (Berlin, 21-22 June 2010). In Conference for European Environmental Specimen Banks. Federal Environment Agency (UBA); 2010:24. http://www.norman-network.net/public_docs/slides_berlin_2010/ manuskript_esb_conf_final.pdf.

6. EMPOMAP: Database of Emerging substances - experts, projects, organisations. [http://www.normandata.eu/empomap_index.php?menu_type=1].

7. EMPODAT: Database of geo-referenced monitoring and bio-monitoring dato on emerging substances in air, water and soil. [http://www.normandata.eu/ empodat_index.php?menu_type=2].

8. Norman MassBank. [http://massbank.ufz.de/MassBank]].

9. Schwesig D, Leonards P: NORMAN Protocol for validation of chemical and biological monitoring methods (February 2009). NORMAN; 2009:99. http://www.norman-network.net/public_docs/norman_v1_v2_v3_version_ 02_final_feb2009.pdf.

10. Schwesig D, Borchers U, Chancerelle L, Dulio V, Eriksson U, Farre M, Goksoyr A, Lamoree M, Leonards P, Lepom P, et al: A harmonized European framework for method validation to support research on emerging pollutants. Trac-Trends in Analytical Chemistry 2011, 30:1233-1242.

11. Vrana B, Vermeirssen ELM, Allan IJ, Kohoutek J, Kennedy K, Mills GA, Greenwood R: Passive sampling of emerging pollutants in the aquatic environment: state of the art and perspectives - Position paper. NORMAN; 2009:44. http://www.norman-network.net/public_docs/slides_prague/ norman_position_paper_pas_sampling.pdf.

12. van Leeuwen S, Strub M-P, Cofino W, Lindström G, van Bavel B: 3rd interlaboratory study on perfluorinated compounds in environmental and human matrices. Amsterdam: Institute for Environmental Studies (IVM); 2011:39.

13. Brack W: Effect-Directed Analysis of Complex Environmental Contamination Berlin Heidelberg: Springer; 2011.

14. Horai H, Arita M, Kanaya S, Nihei Y, Ikeda T, Suwa K, Ojima Y, Tanaka K, Tanaka S, Aoshima K, et al: MassBank: a public repository for sharing mass spectral data for life sciences. Journal of Mass Spectrometry 2010, 45:703-714.

15. Schulze T, Schymanski E, Stravs M, Neumann S, Krauss M, Singer H, Hug C, Gallampois C, Hollender J, Slobodnik J, Brack W: NORMAN MassBank. Towards a community-driven, open-access accurate mass spectral database for the identification of emerging pollutants. NORMAN Network Bulletin; 2012:9-10. http://www.norman-network.net/newsletters/ newsletter_norman_3a.pdf.

16. Hildebrandt C, Wolf W, Neumann S: Database supported search for metabolite identification. Bioinformatics: Journal of Integrativr; 2011:8.

doi:10.1186/2190-4715-24-29

Cite this article as: Brack et al:: The NORMAN Network and its activities on emerging environmental substances with a focus on effect-directed analysis of complex environmental contamination. Environmental Sciences Europe 2012 24:29.

\section{Submit your manuscript to a SpringerOpen ${ }^{\odot}$ journal and benefit from:}

- Convenient online submission

- Rigorous peer review

- Immediate publication on acceptance

- Open access: articles freely available online

- High visibility within the field

- Retaining the copyright to your article

Submit your next manuscript at $>$ springeropen.com 